\begin{tabular}{|c|c|c|}
\hline Beitr. Ent. & Keltern & ISSN 0005-805X \\
\hline $\mathbf{5 4}(2004) 1$ & S. $97-105$ & 2004 \\
\hline
\end{tabular}

\title{
Hymenoptera collections from Qatar, the United Arab Emirates and Oman
}

D. B. BAKER

\section{Summary}

J. N. B. Brown, I. L. Hamer and C. G. Roche made important collections of Hymenoptera (Sphecoidea and Apoidea) in Qatar, the United Arab Emirates and Oman between 1979 and 1993. An annotated list of their collecting stations is provided in order to facilitate future use of their material.

\section{Zusammenfassung}

In den Jahren zwischen 1979 und 1993 haben J. N. B. Brown, I. L. HAMER und C. G. Roche in Katar, den Vereinigten Arabischen Emiraten und in Oman bedeutende Hymenopteren-Kollektionen (Sphecoidea und Apoidea) zusammengetragen. Hier werden Angaben zu den Fundplätzen und dem Sammlungsverbleib kommentiert, um die künftige Auswertung ihres Materials zu erleichtern.

\section{Key words}

Persian Gulf - biodiversity - collectors - deposition of collections

Introduction

Extensive collections of, chiefly, aculeate Hymenoptera, were made in Qatar, in the United Arab Emirates and in Oman by the late J. N. B. Brown, by I. L. Hamer and by C. G. Roche while resident in the Gulf. These collections, which comprise some thousands of specimens, constitute an unique and important source of material for work on the regional Sphecoidea and Apoidea. Since they either have been, or it is intended that ultimately they should be, deposited in institutional collections (material held by the present author in the Division of Entomology, University of Kansas, Lawrence), it would appear desirable to facilitate their future use by amplifying the labelling of the specimens. The material as a whole is unusually well prepared and in very clean condition. The Apoidea in these collections have for the greater part been determined and recorded by the present author, but a considerable proportion of the species represented is as yet either undescribed or unpublished. Apart from the above three collectors, the late K. M. G. Guichard made small collections on short visits to Oman and Saudi Arabia, while E. A. Sugden made collections, including trap-nest collections, at Dubai in 1984 and 1985. No complete listing of GuicharD's localities has been obtainable; his Apoidea are for the greater part in London. Sugden's Apoidea, including Megachilidae determined by the present author, are in the University of California collection at Davis. 


\section{J. N. B. BROWN}

JoHn (but always known as 'Bish') BRown collected in UAE and Oman between 1981 and 1992. His collection is dispersed, being distributed between the HAMER and Roche collections, with some duplicates in the present author's collection. Dathe (2000: 169) described the remarkable hylaeid, Hylaeus emir, from an unique male collected by Brown at Jabāl Ḩafit in UAE.

\section{L. HAMER}

IAN Hamer collected in the Gulf States between 1981 and 1993. His personal collection has been deposited in the Natural History Museum, London; duplicates of his Sphecoidea are in Roche; duplicates of his Apoidea, and some material awaiting publication, are held by the present author. New bees collected by HAMER included two Systropha (BAKER, 1996) and remarkable Chalicodoma and Ammobatini, the latter including a minute cleptoparasite of Nomioides, Hameria nearchus (Baker, in press, and see Michener, 200o: 643, under Parammobatodes Popov, 1931).

\section{G. ROCHE}

GILEs Roche collected in the Gulf States between 1979 and 1982. His personal collection is temporarily housed in the Maidstone Museum, Kent; duplicates of his Apoidea, and some material awaiting publication, are held by the present author. Roche's Sphecoidea will ultimately be deposited in the California Academy of Sciences collection, his Apoidea in the Oxford University Museum of Natural History, Oxford. All specimens collected or received by Roche were serially numbered and a catalogue is maintained by him. New bees collected by Roche included Pseudapis stenotarsus (BAKER, 2002).

\section{List of Localities}

The list given below covers all places in Qatar, the UAE and Oman where collections are known to have been made, but may not be exhaustive, especially for localities visited by BRown. For localities visited by HAMER and by Roche, the list is based on information provided by them, supplemented by the evidence of pin labels observed by the present author; for BROwn, the list rests primarily on the evidence of pin labels.

The first column gives place-names as they appear on pin labels. The great majority of these will not be found in maps at atlas scales, and many neither in larger scale maps nor in standard gazetteers. The names used by the collectors were largely derived from road signs or other local sources, such as oil companies' maps, and may not represent rigorous transcriptions, following any generally recognized system, from the Arabic. In the few instances where the original Arabic spelling has been ascertained, this has been given, transcribed in accordance with the BGN/PCGN system, in square brackets, following the label spelling.

The second column gives geographical coordinates for the localities. Those for his own and BRown's localities were for the most part supplied by I. L. HAMER; others were in part derived from a copy (annotated by Hamer with the numbers of his collecting sites) of the 1:500,000 United Arab Emirates map compiled and drawn by the Survey and Cartography Division of the British Petroleum Co Ltd (September 1975 revised August 1982), in part from other sources including the Microsoft ${ }^{\text {TM }}$ Encarta World Atlas (1988 Edition). It will be noted that in some instances two sets of coordinates are given for one named locality: localities known by the collectors under the same name, while in the same general area, may not be strictly identical. Eastings are given first.

The third column indicates the names of those who made collections at the site in question $(\mathrm{B}=$ Brown; $\mathrm{H}=$ HAMER [followed by his site number, or, if not numbered on map, a known date of visit]; $\mathrm{R}$ = Roche). Comments on localities, given immediately following these indications, were, unless otherwise noted, supplied by Roche. 


\begin{tabular}{|c|c|c|}
\hline \multicolumn{3}{|l|}{ QATAR } \\
\hline Al Khor [Al Khawr] & $\begin{array}{l}51^{\circ} 30^{\prime} \\
25^{\circ} 41^{\prime}\end{array}$ & R. 'Town on east coast north of Doha.' \\
\hline Al Khor district & $-\quad-$ & $\begin{array}{l}\text { R. 'I collected in a farm a few miles north of } \mathrm{Al} \text { Khor on } \\
\text { the east coast.' }\end{array}$ \\
\hline $\begin{array}{l}\text { Al Shahaniyeh } \\
\text { [Ash Shaḩaniyah] }\end{array}$ & $\begin{array}{l}51^{\circ} 14^{\prime} \\
25^{\circ} 22^{\prime}\end{array}$ & $\begin{array}{l}\text { R. 'A water-well area in the centre of Qatar about } 30 \mathrm{~km} \\
\text { WNW of Doha. It is quite extensive and well irrigated, } \\
\text { planted with various vegetable crops as well as natural sc- } \\
\text { rub plants, Acacia, Mimosa, heliotrope etc. It also houses } \\
\text { half the herd of Arabian oryx formed by the late Sheikh } \\
\text { Jassin bin Hamad al Thani, the largest herd in the world.' }\end{array}$ \\
\hline Al Sinnah & $?$ ? & $\begin{array}{l}\text { R. 'Small village to north of Al Shahaniyeh.' 'A group } \\
\text { of three small planted areas about } 45 \mathrm{~km} \text { northwest of } \\
\text { Doha. [An adventitious plant community had deve-loped] } \\
\text { around irrigated Mimosa. It did not last long; it seemed as } \\
\text { though the irrigation was discontinued.' }\end{array}$ \\
\hline Doha [Ad Dawḩah] & $\begin{array}{l}51^{\circ} 36^{\prime} \\
25^{\circ} 15^{\prime}\end{array}$ & R. 'This refers to gardens of private houses in the capital.' \\
\hline Umm al Shahkut & $?$ ? & R. 'A farm north-west of Al Khor.' \\
\hline Umm Ghain & ? ? & R. 'A farm north-west of Al Khor.' \\
\hline
\end{tabular}

\section{UAE}

\begin{tabular}{|c|c|}
\hline Abu Dhabi $[A b u ̄$ & $54^{\circ} 22^{\prime}$ \\
\hline
\end{tabular}

Abu Dhabi (SF) _ _ $\quad$ R. 'Abu Dhabi sewage farm. Plenty of water and vegetation.'

Abu Dhabi (BE)

R. 'The grounds of the British Embassy in Abu Dhabi city. ... one of the few pieces of ground on Abu Dhabi which has remained undisturbed for years. Tamarisks and Zygophyllum sp. were the most attractive plants.' 


\begin{tabular}{|c|c|c|}
\hline Abu Dhabi & $-\quad-$ & $\begin{array}{l}\text { R. 'Abu Dhabi without a detailed place in brackets re- } \\
\text { fers to roadside collecting on heliotrope and Zygo-phyl- } \\
\text { lum with the occasional acacia tree.' }\end{array}$ \\
\hline Abu Samra & $55^{\circ} 24^{\prime} 24^{\circ} 12^{\prime}$ & $\mathrm{B}$ \\
\hline \multicolumn{3}{|l|}{ Adam: see Bid'al'Ajam } \\
\hline \multicolumn{3}{|l|}{ Adhan: see Idhn } \\
\hline Al Ain [Al-'Ain] & $55^{\circ} 44^{\prime} 24^{\circ} 15^{\prime}$ & $\mathrm{H} 24$ \\
\hline Al Ain (I'con) & - & $\mathrm{H}$ [Grounds of Intercontinental Hotel] \\
\hline Al Ain road & - & $\mathrm{B}$ \\
\hline Al Awir / Mileiha & $\begin{array}{l}\text { between } 55^{\circ} 33^{\prime} \\
25^{\circ} 12^{\prime} \& 55^{\circ} 54^{\prime} \\
25^{\circ} 12^{\prime}\end{array}$ & H(1 v 1987). 'Sand dunes' \\
\hline Al Babha & $? \quad ?$ & $\begin{array}{l}\text { R. 'A large plantation on the road towards Liwa [the } \\
\text { Liwa depression] to the southwest of Abu Dhabi. The } \\
\text { plantation was mostly of Mimosa and Acacia with the } \\
\text { usual Zygophyllum and heliotrope.' 'Between Habshān } \\
\text { and Medinat Zaid.' }\end{array}$ \\
\hline $\begin{array}{l}\text { Al Futaisi I. (or } \\
\text { Futaisi) }\end{array}$ & $54^{\circ} 20^{\prime} 24^{\circ} 22^{\prime}$ & $\begin{array}{l}\text { H37, R. 'An island to the southwest of Abu Dhabi. } \\
\text { Mainly scrub with acacias. }\end{array}$ \\
\hline $\begin{array}{l}\text { 'Al Hayl (or Hayl) } \\
\text { [Hayl] }\end{array}$ & $56^{\circ} 14^{\prime} 25^{\circ} 04^{\prime}$ & $\mathrm{H} 13$ \\
\hline $\begin{array}{l}\text { Al Markhaniyah (or } \\
\text { Markheniya), nr. Al } \\
\text { Ain [Al 'Ayn] }\end{array}$ & $55^{\circ} 36^{\prime} 24^{\circ} 12^{\prime}$ & $\begin{array}{l}\text { B, H23, R. 'A large plantation area which I only dis- } \\
\text { covered near the end of my stay in UAE. Until it got } \\
\text { flooded in the rains of February } 1982 \text {, it was a very } \\
\text { interesting place. Most of my collecting was done on } \\
\text { Zygophyllum and heliotrope (as usual). It is only } 15 \mathrm{~km} \\
\text { from Al Saad (and from Al Ain) but it seemed to have a } \\
\text { quite different and wider fauna.' }\end{array}$ \\
\hline Al Saad & $55^{\circ} 28^{\prime} 24^{\circ} 12^{\prime}$ & $\begin{array}{l}\mathrm{H} 22 \text {, R. 'A roadside locality about } 25 \mathrm{~km} \text { west of Al } \\
\text { Ain. Acacia, Zygophyllum and heliotrope irrigated and } \\
\text { further enriched by Baluchi lorry drivers who stopped } \\
\text { at a Coca Cola stall across the road.' 'Abu Dhabi-A'in } \\
\text { road } 100 \mathrm{~km} \text { E of junction with Dubai - Tarif road.' }\end{array}$ \\
\hline $\begin{array}{l}\text { Asimah (or Wadi Asi- } \\
\text { mah near Tayidah) }\end{array}$ & $56^{\circ} 06^{\prime} 25^{\circ} 25^{\prime}$ & $\mathrm{H} 8, \mathrm{R}$ \\
\hline
\end{tabular}




\begin{tabular}{|c|c|c|}
\hline Badayah & $56^{\circ} 21^{\prime} 25^{\circ} 27^{\prime}$ & B. \\
\hline Bid' al' Ajam (Adam) & $55^{\circ} 15^{\prime} 24^{\circ} 11^{\prime}$ & $\begin{array}{l}\text { R. A 'village on the Abu Dhabi - Al Ain road. An area of trees by } \\
\text { the road.' '72km E of junction with Dubai - Tarif road.' }\end{array}$ \\
\hline Bithnah & $56^{\circ} 14^{\prime} 25^{\circ} 10^{\prime}$ & $\mathrm{H} 12$ \\
\hline Das (island) [Dās] & $52^{\circ} 52^{\prime} 25^{\circ} 09^{\prime}$ & H39 \\
\hline Dibba [Dabā] & $56^{\circ} 15^{\prime} 25^{\circ} 37^{\prime}$ & $\mathrm{H} 3, \mathrm{R}$ \\
\hline $\begin{array}{l}\text { Dhaid (or Al Dhaid) } \\
\text { [Adh Dhayd] }\end{array}$ & $55^{\circ} 53^{\prime} 25^{\circ} 17^{\prime}$ & R. 'Similar to Mileiha' [q.v.]. 'A town in the east of the country.' \\
\hline Dhaid-Mileiha road & ? ? & $\begin{array}{l}\text { R. 'A farm which proved quite useful collecting at the edges of } \\
\text { the crops.' }\end{array}$ \\
\hline Dibayah (Zubayah) & $54^{\circ} 08^{\prime} 24^{\circ} 19^{\prime}$ & B. \\
\hline $\begin{array}{l}\text { Diqdaqa (Digdaga) } \\
\text { [Diqdaqah] }\end{array}$ & $55^{\circ} 59^{\prime} 25^{\circ} 40^{\prime}$ & $\begin{array}{l}\text { B, R. 'Rough scrub about } 20 \mathrm{~km} \mathrm{~S} \text { of Ras al Khaimah.' 'Wayside } \\
\text { collecting near the vllage.' }\end{array}$ \\
\hline Dubai [Dubayy] & $55^{\circ} 18^{\prime} 25^{\circ} 15^{\prime}$ & $\mathrm{H} 33$ \\
\hline Falaj al Mualla & $55^{\circ} 44^{\prime} 25^{\circ} 21^{\prime}$ & B. \\
\hline Fagsha & $56^{\circ} 11^{\prime} 25^{\circ} 08^{\prime}$ & H. [Species-rich site, as noted by the collector]. \\
\hline Ghayl-Munay & ca. $56^{\circ} 04^{\prime} 25^{\circ} 24^{\prime}$ & B. \\
\hline Ghosam & $56^{\circ} 09^{\prime} 25^{\circ} 35^{\prime}$ & $\mathrm{H} 5$ \\
\hline Hatta & $-\quad-$ & $\begin{array}{l}\text { R. 'A wadi in the foothills near the Omani border SW of Fujeirah. } \\
\text { [Al Fujayrah] There are gardens there but I collected only outside } \\
\text { them. The fauna tended to be markedly different from that of the } \\
\text { lowlands.' [Many of the bees collected at Hatta were, in fact, not } \\
\text { collec-ted elsewhere, bearing out the collector's comment.] 'Town } \\
\text { with a spring which caused a lot of vegetation.' }\end{array}$ \\
\hline Hatta & $56^{\circ} 09^{\prime} 24^{\circ} 48^{\prime}$ & B, H18 \\
\hline Hatta-Madam road & $? \quad ?$ & R. 'Scrub, roadside collecting area. Not particularly fruitful.' \\
\hline Hili [Hilli] & $55^{\circ} 48^{\prime} 24^{\circ} 18^{\prime}$ & $\mathrm{H} 25$ \\
\hline Huwailat [Howaylat] & $56^{\circ} 11^{\prime} 24^{\circ} 52^{\prime}$ & H17 \\
\hline
\end{tabular}




\begin{tabular}{|c|c|c|}
\hline $\begin{array}{l}\text { Idhn Dibba [= Idhn to } \\
\text { Dibba] }\end{array}$ & $\begin{array}{l}\text { from } 56^{\circ} 01^{\prime} 25^{\circ} 27^{\prime} \\
\text { to } 56^{\circ} 15^{\prime} 25^{\circ} 36^{\prime}\end{array}$ & $\begin{array}{l}\text { B. 'Idhn is a small village } 25 \text { miles south of Ras al Khaimah. } \\
\text { The BP map spells it as Adhan' [ILH]. Cf. Uyay-nah. }\end{array}$ \\
\hline Jebal Ali [Jabal 'Alī] & $55^{\circ} 06^{\prime} 25^{\circ} 02^{\prime}$ & H34 \\
\hline Jebal Dhana [Az̧ Zannah] & $52^{\circ} 39^{\prime} 24^{\circ} 10^{\prime}$ & $\mathrm{H} 38$ \\
\hline Jabal Hafit [Jabāl Ḩafit] & $55^{\circ} 46^{\prime} 24^{\circ} 06^{\prime}$ & B. \\
\hline Jebel Qatar & $55^{\circ} 55^{\prime} 24^{\circ} 21^{\prime}$ & H(6 xi 1987) \\
\hline Khatt [Al Khațț] & $56^{\circ} 01^{\prime} 25^{\circ} 37^{\prime}$ & B. \\
\hline Khor Fakkan [Khawr Fakkān] & $56^{\circ} 20^{\prime} 25^{\circ} 21^{\prime}$ & $\mathrm{B}, \mathrm{H} 11, \mathrm{R}$ \\
\hline $\begin{array}{l}\text { Khor Kalba (or Kalba) } \\
\text { [Khawr Kalbah] }\end{array}$ & $56^{\circ} 21^{\prime} 25^{\circ} 02^{\prime}$ & B, H14 \\
\hline Leyahlia & $?$ ? & R. 'A new plantation area east of the Liwa road. Not fruitful.' \\
\hline $\begin{array}{l}\text { Liwa (oases) }[\mathrm{Al} \\
\text { Jiwā] }\end{array}$ & $\begin{array}{l}\text { from ca. } 53^{\circ} 35^{\prime} \\
23^{\circ} 05^{\prime} \text { to ca. } \\
54^{\circ} 02^{\prime} 23^{\circ} 05^{\prime}\end{array}$ & $\mathrm{H} 4$ \\
\hline Madam (Al Madam) & $55^{\circ} 48^{\prime} 24^{\circ} 59^{\prime}$ & B, H19 \\
\hline Masafi [Masāfī & $56^{\circ} 08^{\prime} 25^{\circ} 18^{\prime}$ & R. 'A wadi area with water and lush vegetation.' \\
\hline Mileiha & $?$ ? & $\begin{array}{l}\text { R. 'Calotropis procera by the roadside about } 20 \mathrm{~km} \text { south } \\
\text { of Dhaid. [Calotropis procera Ait., AsCLEPIA-DACEAE, } \\
\text { French cotton.] }\end{array}$ \\
\hline Munai [Munay'i] & $56^{\circ} 13^{\prime} 24^{\circ} 55^{\prime}$ & $\mathrm{H} 16$ \\
\hline Rifaa & $55^{\circ} 46^{\prime} 25^{\circ} 44^{\prime}$ & \\
\hline Ras al Gharab & $54^{\circ} 30^{\prime} 24^{\circ} 37^{\prime}$ & B. 'E of Sadiyat I.' \\
\hline Remah & $55^{\circ} 19^{\prime} 24^{\circ} 11^{\prime}$ & H (10 iv 1988) \\
\hline Sadiyat I. & $54^{\circ} 24^{\prime} 24^{\circ} 32^{\prime}$ & B. \\
\hline Sha'am & $56^{\circ} 06^{\prime} 26^{\circ} 03^{\prime}$ & \\
\hline Shweib & $55^{\circ} 50^{\prime} 24^{\circ} 47^{\prime}$ & $\mathrm{H} 20$ \\
\hline Shweib-Madam road & ca. $55^{\circ} 40^{\prime} 4^{\circ} 55^{\prime}$ & $\begin{array}{l}\text { B, R. 'This was a collecting site by the roadside mainly } \\
\text { on Calotropis bushes in flower. The road is between Al } \\
\text { Ain and Hatta, perhaps } 50 \mathrm{~km} \text { north of Al Ain. I found } \\
\text { it a very profitable place.' }\end{array}$ \\
\hline
\end{tabular}




\begin{tabular}{|c|c|c|}
\hline Sila / J. Dhana [coast road between] & $\begin{array}{l}\text { between } \\
52^{\circ} 30^{\prime} 24^{\circ} 07^{\prime} \\
\& 51^{\circ} 09^{\prime} 24^{\circ} 36^{\prime}\end{array}$ & B. \\
\hline Site 1 (or Al Ain road Site 1 ) & $\begin{array}{l}\text { ca } 54^{\circ} 50^{\prime} \\
24^{\circ} 13^{\prime}\end{array}$ & B. [? well no. 1 of BP map at $54^{\circ} 42^{\prime} \mathrm{E} 24^{\circ} 14^{\prime} \mathrm{N}$ ] \\
\hline Suleimat [As Sulaymāt] flyover & $55^{\circ} 36^{\prime} 24^{\circ} 11^{\prime}$ & B. \\
\hline $\begin{array}{l}\text { Suweihan (or Sweihan } \\
\text { road)[Țawī Suweihan] }\end{array}$ & $55^{\circ} 19^{\prime} 24^{\circ} 29^{\prime}$ & $\begin{array}{l}\text { B, H21, R. 'A small area of mixed flowering plants } \\
\text { and Zygophyllum about } 40 \mathrm{~km} \text { east of Abu Dhabi.' }\end{array}$ \\
\hline Towean / Dibba & $\begin{array}{l}\text { between } \\
56^{\circ} 02^{\prime} 25^{\circ} 33^{\prime} \\
\& 56^{\circ} 15^{\prime} 25^{\circ} 37^{\prime}\end{array}$ & $\mathrm{H}(22$ iv 1988$)$ \\
\hline Umm al Nar (or Umm an Nar) & $54^{\circ} 29^{\prime} 24^{\circ} 26^{\prime}$ & B. \\
\hline $\begin{array}{l}\text { Uyaynah (or Wadi Uyaynah) [Al } \\
\text { Uyaynah] }\end{array}$ & $56^{\circ} 09^{\prime} 25^{\circ} 31^{\prime}$ & $\begin{array}{l}\text { H6, R. 'A steep road leading down to Dibba on } \\
\text { theas coast from Masafi. Water running down the } \\
\text { road and fairly lush vegetation on either side.' }\end{array}$ \\
\hline Uyaynah (or Wadi Uyaynah) & $56^{\circ} 10^{\prime} 25^{\circ} 28^{\prime}$ & B. \\
\hline Wadi Bih (or Bihr) [Wādī Bih] & $56^{\circ} 06^{\prime} 25^{\circ} 47^{\prime}$ & B, H1 \\
\hline Wadi Fey (or Fay) & $56^{\circ} 11^{\prime} 25^{\circ} 37^{\prime}$ & $\begin{array}{l}\text { H4, R. 'A wadi over the hills at the back of Dibba. } \\
\text { Less lush than Wadi Uyaynah.' }\end{array}$ \\
\hline Wadi Huwaylat [Huwaylāt] & $56^{\circ} 10^{\prime} 24^{\circ} 53^{\prime}$ & B. \\
\hline Wadi Sifuni & $56^{\circ} 00^{\prime} 25^{\circ} 12^{\prime}$ & B. \\
\hline Wadi Sidr [Wādī Sidr] & $56^{\circ} 07^{\prime} 25^{\circ} 27^{\prime}$ & $\mathrm{H} 7$ \\
\hline Worayah & $56^{\circ} 16^{\prime} 25^{\circ} 23^{\prime}$ & $\mathrm{H} 9$ \\
\hline Wahlah [Waḩlah] & $56^{\circ} 17^{\prime} 24^{\circ} 56^{\prime}$ & H15 \\
\hline Zibara & $54^{\circ} 40^{\prime} 24^{\circ} 38^{\prime}$ & $\mathrm{H} 35$ \\
\hline Zubarah & $56^{\circ} 20^{\prime} 25^{\circ} 16^{\prime}$ & $\mathrm{H} 10$ \\
\hline
\end{tabular}




\section{OMAN}

Roche noted of his localities: 'Whilst technically in the Oman, none of the localities at which I collected were as far as the frontier posts which are often 20 or more kilometres inside'.

Abool [A'bal]

Ain Razat

Al Hamra $[\mathrm{Al}$

Ḩamrā']

$\mathrm{Al}$ Khașab

Al Madha

Al Qabil

Buraimi $[\mathrm{Al} \mathrm{Bu}-$

raymī]

Jebal Hawrah [Jabal

Hawrah]

Madabah (or Mad-habbah) [Maḑabbah]

Madabah

Mahdah [Maḩḑah]

Musah [Masah]

Rayy

Rustaq

Sayh Huwayyah (or S Huwayyah or Sih Huwayyah) [Shuwayyah] $56^{\circ} 03^{\prime} 24^{\circ} 25^{\prime}$

$53^{\circ} 59^{\prime} 16^{\circ} 56^{\prime}$

$57^{\circ} 12^{\prime} 23^{\circ} 04^{\prime}$

$56^{\circ} 1526^{\circ} 14^{\prime}$

$56^{\circ} 15^{\prime} 25^{\circ} 16^{\prime}$

$55^{\circ} 49^{\prime} 23^{\circ} 56^{\prime}$

$55^{\circ} 45^{\prime} 24^{\circ} 15^{\prime}$

$55^{\circ} 53^{\prime} 24^{\circ} 20^{\prime}$

$56^{\circ} 09^{\prime} 24^{\circ} 05^{\prime}$

$56^{\circ} 07^{\prime} 24^{\circ} 05^{\prime}$

$55^{\circ} 58^{\prime} 24^{\circ} 24^{\prime}$

$56^{\circ} 04^{\prime} 24^{\circ} 22^{\prime}$

$56^{\circ} 07^{\prime} 24^{\circ} 39^{\prime}$

$57^{\circ} 25^{\prime} 23^{\circ} 26^{\prime}$

$55^{\circ} 52^{\prime} 24^{\circ} 19^{\prime}$
H28. 'In Oman, technically, although reachable from UAE without crossing border posts' [ILH].

H(9 iv 1985). 'Near Salalah' [ILH]

$\mathrm{H}(14$ iv 1985)

H. 'I collected in the Khassab region on one expe-dition' [ILH]. [Extreme north of Oman, on Strait of Hormuz.]

$\mathrm{H}(15$ iv 1985) [S of Ḩafit in UAE]

R 'Oasis town adjoining Al Ain in UAE.'

$\mathrm{H} 26$

H31, R. 'A wadi beyond Buraimi with running water. Plenty of vegetation.'

B.

B, $\mathrm{H} 27$

$\mathrm{H} 29$

$\mathrm{H}(17$ iv 1987)

H (18 iv 1985). 'WSW of Muscat' [ILH].

H (16/18 iv 1987), R. 'A horseshoe-shaped area, surrounded on three sides by hills, east of Buraimi. Mixed scrub vegetation, largely Acacia and Prosopis trees with ground covering of flowers, after rain.' 'Known as Fossil Valley from the abundance of fossils, mainly bivalves. It is about $15 \mathrm{~km}$ by road from $\mathrm{Al}$ Ain in UAE.' [Bees taken here included several species not taken by the collectors elsewhere, including Clavinomia.] 


\begin{tabular}{|c|c|c|}
\hline Wadi Agran & $56^{\circ} 10^{\prime} 24^{\circ} 03^{\prime}$ & $\mathrm{H} 32$ \\
\hline Wadi Fayd, nr Hatta & $? \quad ?$ & $\begin{array}{l}\text { R. 'A wadi beyond Hatta with running water and attendant } \\
\text { vegetation.' }\end{array}$ \\
\hline Wadi Jizi (also Jizzi, Gizi) & $56^{\circ} 10^{\prime} 24^{\circ} 12^{\prime}$ & $\begin{array}{l}\text { H30. 'In Oman, technically, although reachable from UAE } \\
\text { without crossing border posts' [ILH]. }\end{array}$ \\
\hline Wadi Khabb & $56^{\circ} 15^{\prime} 25^{\circ} 46^{\prime}$ & $\begin{array}{l}\text { B, H2. 'Wadi Khabb Shamsi / Wadi Bih was a frequent col- } \\
\text { lecting route' [ILH]. }\end{array}$ \\
\hline $\begin{array}{l}\text { Wadi Khabb (or } \\
\text { Kabb) }\end{array}$ & $56^{\circ} 15^{\prime} 25^{\circ} 40$ & $\begin{array}{l}\text { B. [These coordinates are to approximate mid-point of } \\
\text { Wadi.] }\end{array}$ \\
\hline
\end{tabular}

\section{References}

BAKER, D. B. 1996: Notes on some palaearctic and oriental Systropha, with descriptions of new species and a key to the species (Hymenoptera: Apoidea: Halictidae). - J. nat. Hist. 30: 1527-1547. [Systropha androsthenes, diacantha, spp. nov.]

Baker, D. B. 2002: On Palaearctic and Oriental species of the genera Pseudapis W. F. Kirby, 1900, and Nomiapis Cockerell, 1919 (Hymenoptera, Halictidae, Nomiinae). - Beiträge zur Entomologie, Keltern 52 (1): 1-83. [Pseudapis stenotarsus sp. nov.]

Dathe, H. H. 1995: Studien zur Systematik und Taxonomie der Gattung Hylaeus F. (Apidae, Colletinae). 2. Klärung und Neubeschreibung von Arten arabischer Länder. - Beiträge zur Entomologie, Berlin 45 (1): 155-174. [Hylaeus (Paraprosopis) donbakeri, H. (P.) omanicus, H. (H.) hameri spp. nov.]

Dathe, H. H. 2000: Studien zur Systematik und Taxonomie der Gattung Hylaeus F. (3). Revision der Hylaeusnivalis-Gruppe in Europa und Klärung weiterer westpaläarktischer Arten (Apidae, Colletinae). - Beiträge zur Entomologie, Berlin 50 (1): 151-174. [Hylaeus (Paraprosopis) emir sp. nov.]

Michener, C. D. 2000: The bees of the World. Pp. [i]-xiv, 1-913, 16 pll. Baltimore and London; John Hopkins University Press.

\section{Author's address:}

\section{Dr. D. B. BaKeR}

Hope Entomological Collections

University Museum of Natural History

Oxford OX1 3PW 